\title{
Artigos
}

\section{Percepciones y expectativas que inciden en la vinculación de los usuarios a los Centros de Atención Psicosocial en Brasil}

\author{
Perceptions and expectations that promote treatment adherence among \\ patients receiving treatment at Psychosocial Care Centers in Brazil (abstract: p. 14) \\ Percepções e expectativas que promovem o vínculo dos usuários aos \\ Centros de Atenção Psicossocial no Brasil (resumo: p. 15)

\section{Patricia Elizabeth Sanz de Alvarez ${ }^{(a)}$} \\ $<$ patriciaesanz@gmail.com> iD \\ (a) Secretaria Municipal de Trabalho \\ e Assistência Social, Prefeitura \\ Municipal do Natal. Rua Agnaldo \\ Gurgel Jr, 1840, Casa 4, Candelaria. \\ Nata, RN, Brasil. 59066-030.
}

En el presente estudio se analizan las percepciones y expectativas que promueven la vinculación de los usuarios al tratamiento. Se trata de una investigación cualitativa, de cuño descriptivo e interpretativo que forma parte de una tesis doctoral realizada con 44 usuarios atendidos en cuatro Centros de Atención Psicosocial (CAPS) de un municipio de Rio Grande del Norte, Brasil. Los datos fueron obtenidos por entrevistas y grupos focales, sistematizados con el software QDA Miner LiteV1.4.3, aplicada la técnica de análisis temático y discutidos a partir de referencial teórico del campo de la salud mental. Los principales hallazgos apuntan a transformaciones positivas en sus vidas, el miedo de las internaciones, la concentración del cuidado en un único servicio y mantener la calidad de vida alcanzada. La investigación permitió identificar factores de protección para la efectiva vinculación de los usuarios a los CAPS.

Palabras clave: Tratamiento. Servicios de salud mental. Factores de protección. 


\section{Introducción}

En el marco del trabajo en salud mental, posterior a la Reforma Psiquiátrica, y a partir de experiencias consolidadas, predominantemente en la Atención Básica, en la Red de Salud Mental se trabaja desde la década del noventa con el concepto de "Cuidado" entendido como la suma de cuidados parciales que constituyen una compleja trama de procedimientos, rutinas, flujos y conocimientos ofrecidos por los cuidadores mediante un proceso dialéctico de complementación, pero también de disputa. Estos autores dejan en claro las tensiones que caracterizan el campo y aportan reflexiones sobre los procesos de trabajo y el cuidado en salud, buscando enriquecer y desarrollar una caja de herramientas para que los gestores y trabajadores puedan enfrentar las situaciones concretas relacionadas a esos temas.

Tal vertiente analítica está vinculada al esfuerzo ético, técnico y político de formulaciones para la innovación de los "modelos técnico-asistenciales" del cuidado en el sistema de salud, que amplían el foco de análisis de los actores y de las fuerzas que intervienen en la implementación de las políticas públicas. En el centro de esa expresión, estaría la observación del modo en que se expresan los acuerdos de saber y de poder en los espacios de cuidado y de la ordenación de la producción de acciones y servicios de salud. Ese abordaje permite articular las prácticas de gestión y de atención al analizar, en el ámbito de los servicios, la relación entre los diferentes actores que protagonizan el sistema de salud, las condiciones de ese protagonismo, las bases normativas y técnicas que sustentan el gerenciamiento y la regulación de los procesos de trabajo y los enunciados de los programas y de las políticas de salud.

El concepto de "Línea de Cuidado" se refiere a una tecnología de organización de la atención formulada en el contexto que analiza la integralidad, particularmente los estudios volcados hacia las prácticas de los servicios y aquellos que buscan caracterizar y analizar el cuidado como un proceso de trabajo. La noción de línea de cuidado es comprendida como una tecnología de gestión de la asistencia capaz de producir niveles avanzados y crecientes de integralidad. Por otra parte, la integralidad como directriz constitucional legal e institucionalmente, es concebida como un conjunto de servicios y acciones de salud, preventivas y curativas, individuales y colectivas, en cada nivel de complejidad del sistema de salud ${ }^{3}$.

Respondiendo a un concepto ampliado de la salud que incluye los determinantes sociales, las políticas públicas en salud mental configuran un campo de actuación caracterizado por la presencia de diversos profesionales del área de salud y de otras áreas del conocimiento con el objetivo común de ofrecer un tratamiento en la perspectiva de la inclusión social. Por lo que resulta un desafío para el Sistema Único de Salud consolidar la integralidad, fortaleciendo y contextualizando las intervenciones psicosociales, puesto que la salud mental propone una mirada que rompa con el modelo hospitalocéntrico que domina los tratamientos y deconstruya el lugar del “enfermo", potencializando su salud y los recursos de que dispone para mantenerla y mejorarla. En la medida en que la perspectiva psicosocial envuelve a la familia, al abordaje interdisciplinario, al trabajo con grupos y a la consideración de la realidad social, la clínica ampliada parece caminar en dirección a tal objetivo ${ }^{4}$.

Los Centros de Atención Psicosocial (CAPS) son instituciones destinadas al cuidado de los pacientes con trastornos mentales, a estimular su integración y reinserción familiar y social, apoyándolos en sus iniciativas de autonomía 5 . Para 
ello ofrecen actividades terapéuticas superando el modelo restricto a las consultas y a la prescripción de medicamentos, pues se trabaja en la perspectiva de "Clínica Ampliada" evitando la reducción del usuario a un simple recorte diagnóstico o burocrático. La Clínica Ampliada representa un instrumento para que los trabajadores y gestores de la salud puedan ver y actuar superando la fragmentación de sus propios conocimientos -favorecido por el sistema de especializaciones- sin dejar de reconocer y de utilizar el potencial de otras áreas del conocimiento. Para ello, es necesario definir las estrategias terapéuticas en esos espacios, reflexionar sobre los conceptos, las prácticas y las relaciones que promueven salud entre las personas: técnicos, usuarios, familiares y comunidad. Todos precisan estar involucrados en esa estrategia, cuestionando y evaluando los rumbos de la clínica y del servicio.

Para la Clínica Ampliada, que busca equilibrar el combate a la dolencia con la producción de vida, el proyecto terapéutico cobra relieve pues organiza el itinerario del cuidado y representa una elaboración adecuada para cada usuario. La atención ofrecida debe ser personalizada de tal manera, que el tratamiento a realizarse -dentro y/o fuera del servicio- sea planificado de acuerdo con sus necesidades individuales y respetando sus particularidades. Un punto que resulta importante destacar sobre el Proyecto Terapéutico Singular (PTS) es que parte de la premisa de que la persona tiene el poder de transformar su relación con la vida y con su propia dolencia. Se considera que el camino a ser recorrido por el usuario es solamente suyo y que será él quien acepte participar, decidiendo cuándo quiere ir, negociando o rechazando las ofertas del equipo de salud ${ }^{7}$.

Pero a pesar de ese gran andamiaje teórico metodológico, persiste el aumento de los problemas en los servicios de Salud Mental para lidiar con los usuarios. Una de las principales quejas de los profesionales trata sobre las dificultades que presentan los pacientes en la adhesión al tratamiento. La no adhesión al tratamiento de pacientes con sufrimiento psicológico, con trastorno mental o consumo abusivo de substancias psicoactivas, repercute de forma negativa sobre las posibilidades de mejoría y aumenta las recidivas e internaciones psiquiátricas ${ }^{8,9}$. Esta situación, que podría estar disminuyendo la calidad de vida de los usuarios y sus familiares e incrementando el gasto público en salud, se configura como un problema de salud pública ${ }^{10}$. Por lo tanto, requiere de las instituciones sanitarias, de las personas con sufrimiento psíquico por trastorno mental, de la familia y de la comunidad en general, seguir aunando esfuerzos en pro de un delineamiento de acciones en el campo de la Salud Mental, coherente con los principios del campo y centrado en las necesidades de las personas afectadas, para contribuir con la calidad de vida y con el bienestar de cada una de ellas.

En un trabajo anterior ${ }^{11}$ en que discutimos sobre la tendencia de investigar la adhesión al tratamiento realizado en los CAPS desde una perspectiva exclusivamente biomédica, nos posicionamos reconociéndola como un fenómeno múltiple y complejo en función de la diversidad de intervenciones que demanda el tratamiento y de la complejidad de los actores sociales que componen dicho escenario. Afirmamos que la adhesión al tratamiento consiste en un proceso de vinculación del usuario, que se inicia en el momento del acogimiento y concluye al desvincularlo del servicio mediante el alta asistida. Proceso que, si bien se relaciona con el usuario, se desarrolla a través de su interacción con otros usuarios, familiares o miembros del equipo, y de la influencia de factores micro y macroestructurales. Cualquier alejamiento del usuario del servicio, ya 
sea por su propia voluntad o por situaciones ajenas a la misma, que genere la ruptura del vínculo establecido y el cese de su participación en las acciones que constituyen el proceso de cuidado ofrecido en la institución, supone la finalización de la adhesión al tratamiento.

Por este motivo resulta necesario identificar los factores que colaboran con la efectiva vinculación de los usuarios a los servicios, que coadyuven en la toma de decisiones y proporcionen un marco de referencia más amplio y específico desde el cual realizar las intervenciones en ese campo. De esta forma, analizaremos a continuación las percepciones y expectativas de los usuarios que favorecen su vinculación al tratamiento realizado.

\section{Metodología}

El trabajo expone resultados parciales de una tesis doctoral que se sustenta en el paradigma cualitativo. Un diseño investigativo de construcción flexible ${ }^{12}$, descriptivo e interpretativo apelando a la triangulación de teorías, métodos y técnicas ${ }^{13,14}$. Efectuamos la investigación en la capital de un estado del noreste brasileño, en los cuatro CAPS de adultos de la ciudad (CAPS III-TM, CAPS II-TM, CAPS III-AD, CAPS II-AD que en lo sucesivo denominaremos Servicios 1, 2, 3 y 4 respectivamente), entre agosto de 2014 y enero de 2015. La captación de los usuarios ocurrió por invitación directa realizada en varias reuniones de grupos terapéuticos en cada servicio. Con los usuarios de cada servicio que aceptaron participar llevamos a cabo entrevistas semiestructuradas individuales y dos grupos focales, uno con usuarios del sexo masculino y otro femenino, valiéndonos de una Guía de entrevistas y otra Guía para grupo foca ${ }^{14}$, ambos instrumentos fueron elaborados para tal finalidad.

Tabla 1. Distribución de los sujetos de la investigación

\begin{tabular}{ccccccc}
\hline & \multicolumn{2}{c}{ Entrevista Individual } & & \multicolumn{2}{c}{ Grupo Focal } \\
\cline { 2 - 3 } & Hombres & Mujeres & & Hombres & Mujeres \\
\hline Servicio 1 & 6 & 6 & 12 & 6 & 6 \\
Servicio 2 & 5 & 7 & 12 & 5 & 7 \\
Servicio 3 & 6 & 4 & 10 & 6 & 4 \\
Servicio 4 & 4 & 6 & 10 & 6 & 6 \\
Total & 21 & 23 & 44 & 23 & 23 \\
\hline
\end{tabular}

Fuente: Datos de la investigación

Grabamos y transcribimos íntegramente los datos que obtuvimos en los grupos y en las entrevistas (ver Tabla 1) conducidas por la primera autora, que se extendieron entre 30 y 60 minutos. Los datos fueron sistematizados con el software QDA Miner LiteV 1.4. $3^{15}$. A continuación, realizamos las tres etapas del análisis de contenido temático ${ }^{16}$ : 1) el análisis previo demandó la lectura fluctuante del corpus comunicacional, la interpretación y organización de los contenidos en núcleos de sentido; 2) la codificación consistió en la identificación de palabras y expresiones que permitieron 
(2)

organizar las categorías y 3) el análisis final de los resultados con la lectura de textos teóricos pertinentes.

El Comité de Ética de la Universidad Federal de Rio Grande del Norte aprobó el proyecto bajo el dictamen 510825/2014.

\section{Resultados y discusión}

Del proceso de codificación del corpus de informaciones y después de separar los bloques temáticos coincidentes identificamos las siguientes categorías: la fe, el miedo provocado por la vivencia manicomial, el vínculo con el equipo, las implicaciones del diagnóstico, el tratamiento y los cambios operados. Además, entre las expectativas identificamos: la reinserción social/familiar, la calidad de vida, y la esperanza.

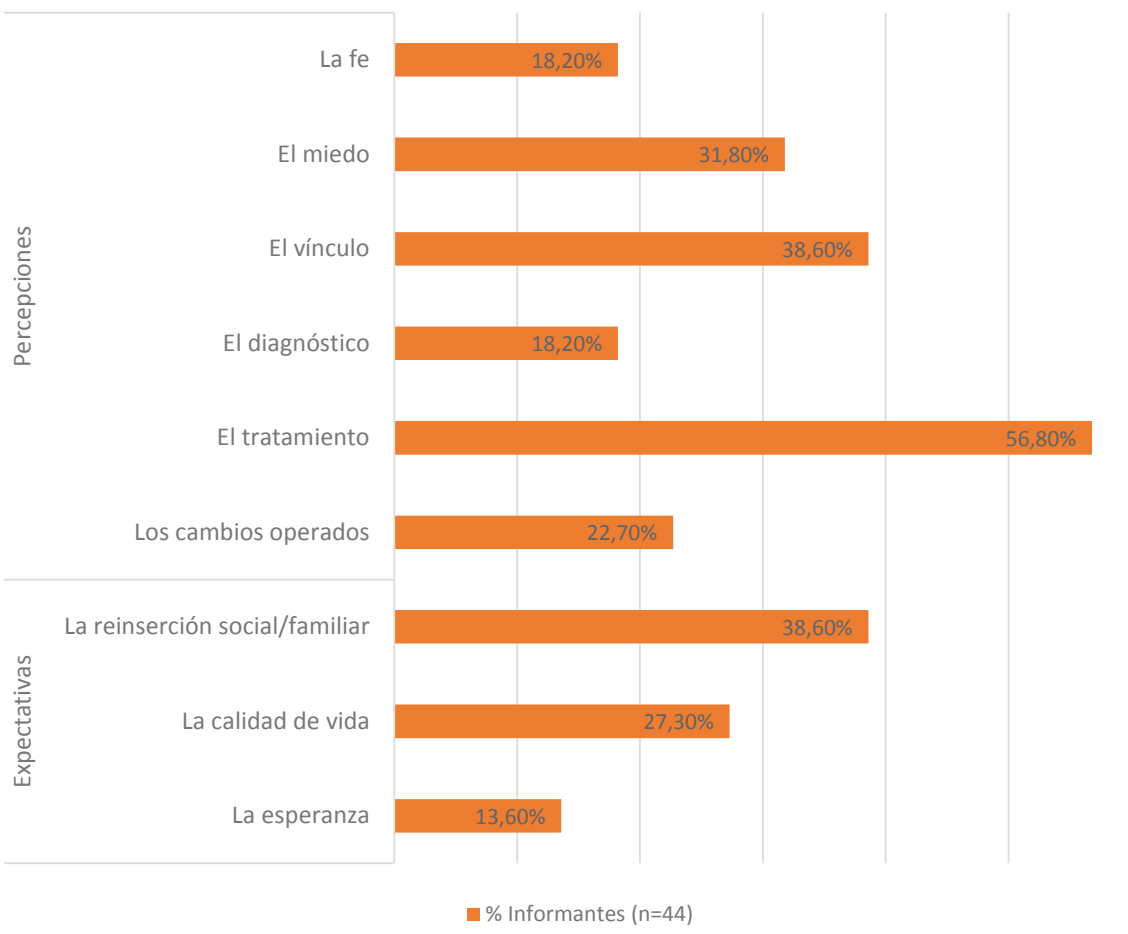

Gráfico 1. Percepciones y expectativas de los usuarios sobre el tratamiento realizado

Fuente: Resultados de la investigación QDA Miner.

Las percepciones se refieren al significado atribuido por los usuarios a diversas experiencias objetivas y subjetivas adquiridas a lo largo de su vida. Presentamos las categorías de acuerdo con la frecuencia registrada en el discurso de los entrevistados. Nos referirnos a cada uno de ellos con la letra P seguida por el número de orden con que ingresamos los datos al programa QDA Miner, la inicial correspondiente al sexo (F y M) y el número del Servicio al que pertenece (Por ejemplo: P1/M1). 
(e)

$\mathrm{Al}$ analizar las percepciones y las expectativas de los usuarios que inciden favorablemente en la realización del tratamiento realizado en los CAPS partimos de la premisa de que todos los entrevistados demostraban su adhesión al tratamiento, por el hecho de encontrarse vinculados a los servicios.

\section{Sobre las percepciones y las expectativas}

Muchos entrevistados consideraron que el tratamiento ofrecido en el CAPS era integral por desarrollarse en los turnos mañana y tarde, y por ofrecerles, además de la consulta médica, alimentación, actividades de grupos psicoterapéuticos, terapéutico y operativo, y actividades recreativas y culturales. En otros casos compararon el tratamiento actual con los anteriores realizados en otros contextos y en los que apenas recibían la atención del médico y de una asistente social. Reconocieron que en el CAPS cuentan con una amplia gama de cuidados y que allí pueden estrechar lazos de amistad.

Dónde voy a encontrar un tratamiento así? Allá no teníamos nada de esto [...] tengo los psicólogos que son los que organizan el grupo, eso me hace sentir bien, lástima que es solo 2 veces por semana. También nos divertimos jugando a las damas, al dominó... y hasta me dan de comer. (P.36/M-4)

La mayoría de los entrevistados valoriza el tratamiento realizado en el CAPS, ya que acceden en un único lugar a consultas médicas clínicas y psiquiátricas, acompañamiento psicológico, talleres ocupacionales, recreación, medicamentos gratuitos, alimentación. A lo que se agrega la convivencia y la posibilidad de establecer lazos de amistad con sus pares, que les ofrece cierta tranquilidad. Pero lo que los entrevistados no advirtieron es que la integralidad ${ }^{3}$ del tratamiento ofrecido en el CAPS se apoya en el hecho de contemplar diferentes dimensiones de la vida en el ámbito del cuidado. Contrariamente, identificamos que se restringen a una cierta satisfacción por el hecho de obtener diversas opciones en un mismo servicio.

Otro de los núcleos de sentido identificados en el discurso de los usuarios se refiere al vínculo afectivo que establecen con los profesionales. La percepción de una actitud acogedora, desprovista de prejuicio y con foco en el sufrimiento y en las potencialidades, puede colaborar para que el usuario se reconcilie con sí mismo. Puesto que, en muchos casos, la imagen distorsionada de sí refleja el estigma que continúa asolando a las personas en sufrimiento por trastorno mental y por el abuso de substancias psicoactivas.

El riesgo de sufrir una crisis es algo que genera gran sufrimiento a los usuarios con trastorno mental. Por eso, los profesionales acostumbran a recomendarles que en momentos de crisis recuerden el vínculo que establecieron con ellos y que frente a cualquier situación de emergencia que pueda colocarlos en peligro recurran al servicio, lo que representa una fuente de seguridad. Muchos de los entrevistados del Servicio 1,2 y 4 refieren haberse vinculado con los psicólogos. Sin embargo, puede resultar una falacia pensar que todo psicólogo es empático ya que para algunas de las usuarias del Servicio 3 el vínculo se establece más fácilmente con las médicas, por el hecho de percibir en ellas una mayor demostración de empatía: "Yo me llevo mejor con las médicas, son un amor. Las otras profesionales son bastante antipáticas, bueno... me refiero a que son menos cariñosas" (P.25/F-3). 
Para algunos de los entrevistados, son las pequeñas demostraciones de afecto, un beso o un abrazo, las que consideran importantes. Para otros lo esencial es sentirse cuidados y recibir el apoyo y el soporte emocional de los profesionales, ya que generalmente no cuentan con el apoyo de sus amigos o familiares. El vínculo también parece ser reforzado por la confianza que los profesionales les transmiten, al colaborar con la superación de obstáculos que pudieran presentarse y surge como un elemento central de motivación sobre la fuerza de voluntad y la decisión individual de los entrevistados. El trato amable y desprovisto de prejuicios y es, sin duda, uno de los elementos decisivos para la construcción del vínculo entre profesionales y usuarios. El sentirse tratados de igual a igual por los profesionales del servicio, puede estar relacionado con cuestiones de poder que mantienen las relaciones interpersonales en sociedades de clases como la nuestra:

Para mí el tratamiento es perfecto, no le encuentro errores, tenemos todo lo que precisamos y todos se preocupan con nosotros y nos tratan bien. Sentir que los profesionales no tienen prejuicios conmigo, que me tratan de igual a igual y que siempre buscan entenderme es lo que me da más ganas de mantener mi tratamiento. (P.11/M-1)

La producción de subjetividad, puesta de manifiesto en la cita anterior referida al "trato de igual a igual" que un usuario recibe de los profesionales, nos remite a los desarrollos teóricos de Foucault ${ }^{17}$ y nos permite comprender cómo el cuerpo en la modernidad se instrumenta por medio de técnicas de sujeción y normalización para la conformación de su individualización y su control político. Temas como la sexualidad, higiene, conducta y salud se orientan a construir la individualidad de los sujetos y su ubicación social polarizada, como normal o anormal; sano o enfermo; peligroso o no peligroso. La sujeción a partir de 'aparatos biopolíticos' (como por ejemplo los programas sanitarios, controles inmigratorios, precarización del trabajo, etcétera) establece un orden normativo en el cual se diferencian las 'minorías desviantes', pasibles de persecución, aislamiento, violencia, abandono y hasta eliminación ${ }^{18}$.

Percibimos que la importancia otorgada por los usuarios al vínculo que establecen con los profesionales, más allá de las necesidades individuales de mayores o menores demostraciones de afecto, o cuestiones veladas de poder en las relaciones interpersonales, se traduce en gestos de acogimiento, un trato amable y una actitud de confianza que les permita recuperar su autoestima y mantener sus decisiones. También cobra relevancia ya que impacta positivamente en el desarrollo de su tratamiento.

Los relatos de nuestros entrevistados sobre sus experiencias de internaciones en hospitales psiquiátricos, públicos y privados de la ciudad se encuentran impregnados de historias marcadas por el sufrimiento, el maltrato y la violación de derechos. A continuación, podemos advertir que el miedo es el principal residuo dejado por la experiencia de internación y del cual aún les cuesta librarse: "Es que me muero de miedo que pueda ocurrirme lo que ya me ocurrió, que me internen en el psiquiátrico, por eso ni pienso en dejar mi tratamiento" (P.4/F-1).

Para los usuarios que se encuentran en tratamiento por el uso de substancias psicoactivas la vivencia manicomial, y su consecuente asociación entre manicomio y locura, resulta difícilmente explicable. Muchos se refirieron al sufrimiento padecido 
y cuestionaron la efectividad del tratamiento recibido en el hospital, otros expresaron su preocupación por intentar establecer un límite entre la dependencia química y la locura.

Para mí eso no es un tratamiento, porque te deja peor de lo que vos ya eras. (P.31/M-3)

Sin embargo, la experiencia traumática de haber sido internado en un Hospital Psiquiátrico parece aumentar la percepción de la mayoría de los entrevistados sobre los aspectos positivos que les ha proporcionado el tratamiento realizado en el CAPS, disminuyendo la posibilidad de percibir los aspectos negativos: "Sea como sea, es mil veces preferible el CAPS que el hospital psiquiátrico. Podría pasar la tarde contándole los horrores que vi y que viví ahí. Por eso... cuanto más cerca esté del CAPS más lejos voy a estar del hospital psiquiátrico" (P.3/M-1).

La cita precedente nos remite a considerar que la triste situación de las personas privadas de su libertad debido a su sufrimiento mental corrobora antiguas concepciones de confinamiento y condiciones degradantes a las que resultaron sometidas en muchas de las instituciones psiquiátricas, representando una señal de las violaciones sistemáticas infringidas a los derechos existentes ${ }^{19}$. Consideramos que el miedo provocado por la vivencia manicomial refuerza la decisión de los usuarios a mantenerse vinculados al tratamiento ofrecido en los CAPS.

Otro de los factores identificados se refiere a la percepción de los cambios operados en sus vidas ya que sugiere consubstanciar la decisión de continuar en tratamiento. Los principales cambios destacados por los entrevistados de los Servicios 1 y 2 son: recuperar la voluntad de vivir, adquirir seguridad en sí mismos y desarrollar competencias relacionales. Por su parte, los usuarios de los Servicios 3 y 4 , se refieren al aumento de sus capacidades reflexivas y a la sociabilidad, al manejo de las emociones, la adquisición de autoconfianza y de autoestima, a reanudar los estudios, a la voluntad de trabajar y a la posibilidad de desarrollar un proyecto de vida.

$\mathrm{Al}$ referirse a los cambios operados en sus vidas algunos de los entrevistados destacaron la valorización de la vida. Uno de ellos se refirió al momento crucial en que recibió el diagnóstico de SIDA, corolario de una evaluación clínica realizada en el CAPS y confirmada por los exámenes solicitados posteriormente. Al haberle permitido identificar y tratar una grave enfermedad, el tratamiento realizado en el CAPS le trajo aparejada, como principal cambio, la resignificación del valor de su vida.

Una herramienta científica que tiene la finalidad de clasificar enfermedades por medio de códigos y una variedad de señales, de síntomas y de aspectos considerados anormales es el diagnóstico psiquiátrico, tema que genera polémica y posiciones opuestas. Muchos autores afirman que sirve para rotular a las personas legitimando la tentativa de ejercer un control sobre ellas y otros lo consideran necesario para dar una dirección al tratamiento ${ }^{20}$.

Discusiones mediante, lo cierto es que en el campo de la salud mental brasileña el tratamiento ofrecido a los usuarios se orienta a partir del diagnóstico y comprobamos que la mayoría de los entrevistados conocían sus diagnósticos y sus implicaciones. El recibir del médico la confirmación de la cronicidad del diagnóstico, representa un divisor de aguas en el modo de comprender su propio sufrimiento pues la toma de 
conciencia sobre la irreversibilidad del cuadro parece contribuir con la aceptación de continuar su tratamiento, tal como lo advertimos en la siguiente cita:

Yo estoy haciendo un tratamiento aquí porque mi problema es CIE 10 código 35.1, que viene a ser un trastorno bipolar, es un trastorno mental psicótico con depresión irreversible y trastorno de pánico. Cuando acepté que mi problema es crónico, también acepté que iba a precisar tomar mis remedios, igual que mi marido que es diabético, y así fui aceptando venir acá [al CAPS]. (P.15/F-2)

La autoconciencia y el autocuidado también representan un modo de auto vigilancia para mantener las transformaciones positivas en sus vidas. En una sociedad atravesada y constituida por el biopoder, el autocuidado es regulado por los dictámenes que el modelo biomédico ofrece, ya que al partir de una identificación biológica establece y normativiza formas de vivir ideales ${ }^{21}$. Pero, de modo discrepante, advertimos una menor proporción de entrevistados asistidos por problemáticas asociadas al consumo de alcohol y de otras drogas, que se refieran a cuestiones relacionadas al diagnóstico y a la cronicidad de este. Uno de ellos subraya los resultados obtenidos en las actividades psicoeducativas, las cuales le permitieron comprender que las adicciones precisan ser tratadas de forma continua: "Ahora me vigilo, porque lo más peligroso es cuando uno piensa que está curado" (P.43/M-4).

Entender que las implicaciones del diagnóstico puedan representar un factor de protección colaborando en la vinculación al tratamiento integral de los usuarios, especialmente en el caso de los portadores de trastorno mental, no implica necesariamente dejar de considerar otros análisis procedentes. Ya que también advertimos que a los profesionales les resulta difícil efectivizar el alta de los usuarios, y a los usuarios les resulta difícil aceptar el alta y separarse del servicio, con el consecuente riesgo de institucionalización y el establecimiento de nuevos modos de cronicidad ${ }^{22}$. Pero también identificamos algunas excepciones en las que, si bien la autoconciencia del diagnóstico crónico promueve la adhesión al tratamiento, también puede asociarse con la imposibilidad de mejorar: "Yo sigo al pie de la letra el tratamiento, pero eso de ser paciente crónico no lo termino de digerir" (P.3/M-1).

Es por ese motivo que es necesario estar atentos. Desde el año 2002 Merhy viene alertando sobre el riesgo de institucionalización de los CAPS y de la producción de nuevas cronicidades expresadas por medio de la retención de los usuarios, de los modos de gerenciar resistentes a la implementación de acciones fuera del servicio y de producir puertas de salida y de circulación para la red, corriendo el riesgo de transformar los CAPS en manicomios disfrazados ${ }^{23}$.

Otra de las categorías identificadas es "La fe". Su denominación resulta intencional ya que identificamos en el discurso de nuestros entrevistados diversos tópicos que podrían circunscribirse a la espiritualidad o a la religiosidad, pero que en todos los casos se ven contemplados de un modo genérico, por el término "fe". Gran parte de los entrevistados del Servicio 4, afirman sentirse próximos de un ser superior al que denominan Dios o Jesús y al cual le piden que oriente sus caminos Entre las prácticas realizadas citaron frecuentar la iglesia protestante, establecer nuevas relaciones sociales y vínculos afectivos con las personas que la frecuentan, y su contribución para la 
adhesión al tratamiento: "Estar cerca de Dios me ayuda, él me confirma que no debo abandonar mi tratamiento, porque quiere lo mejor para mí" (P.23/F-2).

También identificamos que la participación en una comunidad religiosa protestante, al parecer destinada a las personas en sufrimiento por el uso de drogas y en situación de calle, parece reforzar la manutención del tratamiento en el CAPS y al mismo tiempo retroalimentarlo. Consideramos que la fe, como experiencia humana cotidiana, amplía la red de apoyo social favoreciendo la adhesión al tratamiento.

A partir de investigaciones realizadas en diversos países europeos algunos autores $^{24}$ afirman que la implicancia religiosa puede aumentar el apoyo social y evitar comportamientos de riesgo como el consumo abusivo de alcohol y otras drogas, inclusive presentan evidencias epidemiológicas sobre una menor prevalencia de esa problemática entre los miembros de iglesias protestantes en Brasil.

También abordamos las expectativas de los usuarios, y en ese caso lo hacemos considerando las creencias que tienen los entrevistados sobre los beneficios que obtuvieron en sus tratamientos, apoyados en sus propias experiencias transformadoras.

Especialmente aquellos entrevistados con mayor tiempo de tratamiento, han logrado percibir que los progresos alcanzados durante el mismo les permitió mejorar el modo de relacionarse con sus familiares y amigos, estableciendo nuevos vínculos, dentro y fuera del servicio, y recomponiendo algunos que parecían fracturados:

A la mayoría de los usuarios como yo, les parece que perdieron los amigos y muchas otras cosas por causa de la enfermedad, pero hoy tengo más amigos que los que tenía antes. (P.3/M-1)

Cuando mi mujer me dejó y me sacaron la patria potestad de mis hijos me di cuenta de que si los quería ver de nuevo precisaba hacer un tratamiento. Hoy consigo ser un buen padre para ellos. (P.37/M-4)

La reinserción social ${ }^{22}$ se refiere a las posibilidades de convivencia del usuario con su prójimo. Esto significa que un día hubo exclusión social y que es necesario devolver el usuario a la matriz social, por medio del contacto con su familia, parejas, amigos y otros miembros de la sociedad, así como la circulación y la ocupación de los espacios sociales. Por lo tanto, uno de los principales objetivos del tratamiento integral ofrecido en los CAPS se refiere a la reinserción social. Observamos que muchos de los usuarios de los Servicios 1, 3 y 4 mantienen la expectativa de conservar y/o aumentar las posibilidades de reinserción familiar y social que concretaron durante el tratamiento.

Uno de los resultados destacados por la mayoría de nuestros entrevistados es la mejoría en la calidad de vida desde que iniciaron el tratamiento en el CAPS, coincidiendo con la literatura ${ }^{24}$ en cuanto a su asociación con el aumento de la espiritualidad discutido no apartado anterior. De hecho, los usuarios mantienen sus expectativas de preservar el bien estar, la paz y las ganas de vivir, dejando en el pasado la depresión y las alucinaciones. Estas fueron algunas de las conquistas que las usuarias manifestaron no querer perder: "Tengo ganas de vivir y de estar cada vez mejor. Espero continuar teniendo salud, sin entrar en crisis, seguir mi tratamiento y estar en paz" (P.12/F-1). 
Las palabras cambio, equilibrio, ganas de vivir, bienestar, rumbo, alegría, fueron algunas de las utilizadas por nuestros entrevistados para referirse a las adquisiciones proporcionadas por el tratamiento realizado. La decisión de no faltar, o el empleo de estrategias que les recuerden el bienestar alcanzado en el trascurso del tratamiento, cuando el desánimo o el desgano pudieran asomarse, son algunas de las experiencias que pueden identificarse en la próxima cita: "Yo dejé el crack gracias a este tratamiento. Aquí me pude equilibrar. El medicamento que me dieron aquí fue santo remedio. ¡Dejé el crack! usted sabe lo que es eso? Y ahora estoy luchando para dejar de tomar, porque quiero tener una vida mejor para mí y para mi hijo especialmente" (P.28/M-3).

Como lo podemos advertir los usuarios otorgan centralidad en el aspecto farmacológico de sus tratamientos. En algunos casos, ocupando un lugar central en la secuencia iniciada por una decisión personal, el remedio y la asistencia diaria al servicio; y dándole a dicha combinación el crédito por los resultados positivos del tratamiento. Por su parte, en la cita anterior, el medicamento es asociado directamente al abandono de una de substancias psicoactivas a la que mantenía su adicción. Pero, en síntesis, en ambos casos el uso del medicamento se presenta como milagroso.

También se vislumbra la reinserción social a través de la convivencia de los entrevistados con personas que enfrentan diversas problemáticas. Dicha convivencia parece favorecer, además de la empatía, la relativización de los propios problemas, con su consiguiente percepción de bienestar:

Desde que estoy aquí mi vida mejoró y no solo mi salud. Me di cuenta de que mis problemas no son tan graves y que tengo condiciones de solucionarlos. Todo eso desde que participo del grupo terapéutico, entre todos aprendemos a encontrar los medios para resolverlos. (P.41/M-4)

Nuestro análisis nos permitió identificar que la mayoría de los usuarios entrevistados percibe una transformación positiva en su vida a lo largo de su tratamiento, la cual les permitió reestablecer lazos familiares y de amistad fracturados, y en algunos casos nuevas amistades. Además de poder relativizar la dimensión de su propia problemática, al confrontarse con el sufrimiento y las problemáticas de otros usuarios. Pero especialmente, advertimos que todos ellos generan expectativas de continuar gozando de la calidad de vida alcanzada por medio del tratamiento realizado en los CAPS.

También identificamos diversos entrevistados que mantienen expectativas sobre la posibilidad de restaurar procesos de trabajos que fueron discontinuados en el servicio. Nos referimos aquí a las marcas y a los registros mnémicos dejados por experiencias de un tiempo pasado. Ocho de los doce entrevistados del Servicio 1 continúan manteniendo su adhesión al tratamiento y vinculados al servicio con la expectativa de que, de un momento a otro, el modo de funcionamiento anterior sea restablecido. Presentamos a seguir una de las citas seleccionadas: "Ya tuvimos tantas cosas en el CAPS... hace más o menos dos años que no tenemos más nada allá, solo la consulta. Yo siempre espero que vuelva a ser como antes" (P.2/F-1).

Como podemos advertir, la mayor parte de los entrevistados atendidos en el Servicio 1 aspira a beneficiarse con alteraciones que podrían ser realizadas en el funcionamiento del servicio y les permitiría recuperar condiciones anteriores de 
tratamiento, que actualmente se caracterizaría por la atención ambulatoria. Aunque las expectativas de los usuarios con mayor tiempo de tratamiento no se basen en hechos concretos, sino en los buenos recuerdos de un servicio que supo ofrecerles un tratamiento integral, las mismas parecen mantenerlos vinculados a pesar de ofrecerles un tratamiento fragmentado.

Cabe entonces preguntarnos si realmente estaríamos frente a un fenómeno de incompletitud institucional, a una cierta satisfacción al recibir todas las formas de cuidado en un único servicio o a un deseo de infantilización de los usuarios de volver a la institución tota ${ }^{25}$ que nada tiene que ver con la autonomía de los sujetos.

\section{Consideraciones finales}

La investigación realizada nos permitió establecer las concepciones presentes en el discurso de los usuarios que representan potenciales factores de protección para su efectiva vinculación al tratamiento realizado en los CAPS. Entre ellos: 1. Considerar la diversificación del cuidado recibido, un tratamiento que supera la consulta médica e incluye la participación en grupos y talleres terapéuticos, además del suministro de la medicación y de alimentación. 2. Establecer un vínculo afectivo con los profesionales del equipo, que en su mayoría se establece con los psicólogos y el caso del Servicio 3, se establece con las médicas, 3 . Haber tenido experiencias traumáticas de internación en hospitales psiquiátricos, 4 . Reconocer los cambios positivos operados en sus vidas desde el inicio del tratamiento, 5 . Considerar la necesidad de su tratamiento para mantener el bienestar debido al alcance de su diagnóstico y 6 . Mantener la fe en la existencia de una entidad superior que vela por su cuidado y le proporciona motivación para soportar los efectos indeseados del tratamiento.

Además, identificamos que los usuarios esperan mantener la calidad de vida alcanzada; mantener, y hasta superar, el estado de equilibrio y el bienestar en las relaciones familiares y sociales y, en el caso del Servicio 1, identificamos el deseo, un tanto ilusorio, de que ocurran transformaciones institucionales que les restituyan un modelo de tratamiento que les fuera ofrecido anteriormente, $\mathrm{y}$ al cual le atribuyen las mejorías alcanzadas.

Cabe destacar que, en una publicación anterior, en la cual caracterizamos el trabajo ofrecido en los CAPS investigados y lo comparamos con las directivas vigentes ${ }^{23}$, advertíamos que en el terreno del cuidado existe un distanciamiento entre la práctica cotidiana de los profesionales y las normativas postuladas. Nos referíamos a la presencia de prácticas que enfatizan las acciones asilares médico-centradas en detrimento de acciones integrales de cuidado potentes, que garanticen el derecho de los usuarios de tener el mejor tratamiento y volcadas para la reinserción social. Pero como lo hemos podido apreciar a lo largo de los resultados exhibidos en el presente estudio, los usuarios entrevistados no parecen percibir dicha situación. 


\section{Agradecimientos}

A la Coordinación de Perfeccionamiento del Personal de Enseñanza Superior - CAPES por la beca de doctorado pleno en el exterior concedida (BEX 9702-13-0)

\section{Derechos de autor}

Este artículo está bajo la Licencia Internacional Creative Commons 4.0, tipo BY (https:// creativecommons.org/licenses/by/4.0/deed.es).

\section{Referencias}

1. Cecílio LCO, Merhy EE. A integralidade do cuidado como eixo da gestão hospitalar. En: Pinheiro R, Mattos RA, organizadores. Construção da integralidade: cotidiano, saberes e práticas em saúde. Rio de Janeiro: IMS, UERJ, Abrasco; 2003. p. 197-210.

2. Merhy EE. A saúde pública como política: um estudo de formuladores de políticas. São Paulo: Hucitec; 1992.

3. Pinto DM, Jorge MSB, Pinto AGA, Vasconcelos MGF, Cavalcante CM, Flores AZT, et al. Projeto terapêutico singular na produção do cuidado integral: uma construção coletiva. Texto Contexto Enferm. 2011; 20(3):493-502.

4. Alves ES, Francisco AL. Ação psicológica em saúde mental: uma abordagem psicossocial. Psicol Cienc Prof. 2009; 29(4):768-79.

5. Brasil. Ministério da Saúde. Secretaria-Executiva. Núcleo Técnico da Política Nacional de Humanização. HumanizaSUS: equipe de referência e apoio matricial. Brasília: Ministério da Saúde; 2004.

6. Campos GWS. A clínica do sujeito: por uma clínica reformulada e ampliada. En: Campos GWS. Saude Paideia. São Paulo: Hucitec; 2003.

7. Brasil. Ministério da Saúde. Portaria 154/GM, de 24 de Fevereiro de 2008. Dispóe sobre a criação dos núcleos de apoio as equipes de saúde da família. Brasília: Ministério da Saúde; 2008.

8. Lew KH, Chang EY, Rajagopalan K, Knoth RL. The effect of medication adherence on health care utilization in bipolar disorder. Manag Care Interface. 2006; 19(9):41-6.

9. Pompili M, Serafini G, Del Casale A, Rigucci S, Innamorati M, Girardi P, et al. Improving adherence in mood disorders: the struggle against relapse, recurrence and suicide risk. Expert Rev Neurother. 2009; 9(7):985-1004.

10. Mari JJ, Jorge MR, Kohn R. Epidemiologia dos transtornos psiquiátricos em adultos. En: Mello MF, Mello AAF, Kohn R, organizadores. Epidemiologia da saúde mental no Brasil. Porto Alegre: ArtMed; 2007. p. 119-41.

11. Alvarez PES, Rosendo E, Alchieri JC. The applicability of the concept of treatment adherence in the context of the Brazilian mental health system. Rev Esc Enferm USP. 2016; 50 Spe:54-60. doi: http://dx.doi.org/10.1590/S0080-623420160000300008.

12. Morse JM. Principles of mixed method and multi-method research design. En: Tashakkori A, Teddlie C. Handbook of mixed methods in social and behavioural research. London: Sage; 2003. 
13. Vasilachis de Gialdino I. Estrategias de investigación cualitativa. Barcelona: Gedisa; 2006.

14. Minayo MCS. El desafío del conocimiento: investigación cualitativa en salud. Buenos Aires: Lugar Editorial; 2004.

15. Hernández ARC. Análise qualitativa usando o programa QDA Miner um exemplo aplicado de pesquisa: análise documental de textos de protesto. En: Scarparo H, organizador. Psicologia e pesquisa: perspectivas metodológicas. 2a ed. Porto Alegre: Sulina; 2008.

16. Minayo MC. Pesquisa social: teoria, método e criatividade. Rio de Janeiro: Vozes; 2009.

17. Foucault M. El poder psiquiátrico. Buenos Aires: Fondo de Cultura Económica; 2007.

18. Galende E, Kraut A. El sufrimiento mental: el poder, la ley y los derechos. Parte I: capítulo IV: el poder psiquiátrico y las razones para su control. Buenos Aires: Lugar Editorial; 2006.

19. Severo AKS, Dimenstein M. O diagnóstico psiquiátrico e a produção de vida em serviços de saúde mental. Estud Psicol. 2009; 14(1):59-67.

20. Menezes FA. Cuidado de si e gestão da vida: da ética grega ao biopoder. Rev Psicol. 2002; 14(2):95-109.

21. Merhy EE. O ato de cuidar: a alma dos serviços de saúde. En: Brasil. Ministério da Saúde. Secretaria de Gestão do Trabalho e Educação na Saúde. Departamento de Gestão da Educação na Saúde. Ver-SUS Brasil: caderno de textos. Brasília: Ministério da Saúde; 2004. p.108-37.

22. Dimenstein M. O desafio da política de saúde mental: a (re)inserção social dos portadores de transtornos mentais. Mental. 2006; 4(6):69-82.

23. Alvarez PES, Rosendo E, Alchieri JC. Centros de Atención Psicosocial: comparación entre el trabajo ofrecido y la legislación. Psicol Estud. 2016; 21(4):653-64.

24. Dalgalarrondo P. Religião, psicopatologia e saúde mental. Porto Alegre: Artmed; 2008. v. 1.

25. Goffman E. Internados. Buenos Aires: Amorrortu editores; 1998.

This study analyses perceptions and expectations that promote treatment adherence. A descriptiveinterpretive study was conducted as part of a doctoral thesis with 44 patients receiving treatment at four psychosocial care centers in a municipality in the State of Rio Grande do Norte, Brazil. Data was collected using interviews and focus groups. Thematic analysis was performed using the software QDA Miner Lite version 1.4.3 and frames of reference from the field of mental health. The findings show that positive transformations occurred in the patients, fear of being admitted to hospital, provision of various care options within the same service, and maintenance of quality of life gains. The study identified protective factors for effective adherence to treatment.

Keywords: Treatment. Mental health services. Protective factors. 
O objetivo deste estudo foi analisar as percepçóes e expectativas que favorecem a vinculação dos usuários ao tratamento. Trata-se de um estudo qualitativo, de caráter descritivo e interpretativo, cujos dados constituem um recorte de tese doutoral, realizado com 44 usuários atendidos em quatro Centros de Atenção Psicossocial (CAPS) de um município do Rio Grande do Norte, Brasil. Os dados foram coletados por entrevistas e grupos focais, sistematizados com o software QDA Miner LiteV 1.4.3, aplicados à técnica de análise temática e discutidos a partir de referenciais teóricos do campo da saúde mental. Os principais achados referem-se às transformaçóes positivas em suas vidas, ao medo de ser internados, ao recebimento de várias opçôes de cuidado no mesmo serviço e à manutenção da qualidade de vida alcançada. A pesquisa permitiu identificar fatores de proteção para a efetiva vinculação dos usuários aos CAPS.

Palavras-chave: Tratamento. Serviços de saúde mental. Fatores de proteção. 